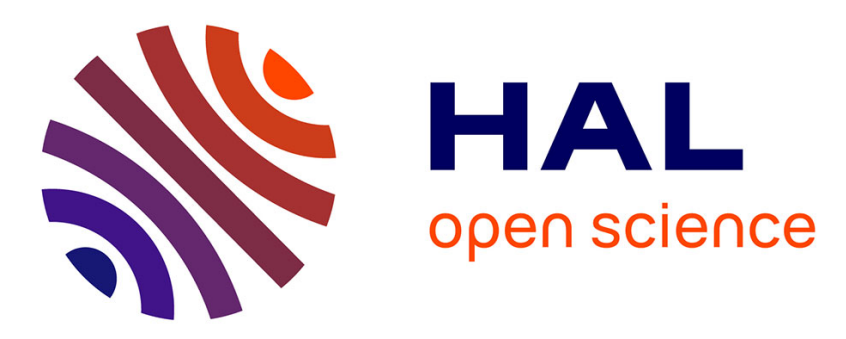

\title{
Automatic Camera Calibration Applied to Medical Endoscopy
}

Joao Barreto, Jose Roquette, Peter Sturm, Fernando Fonseca

\section{To cite this version:}

Joao Barreto, Jose Roquette, Peter Sturm, Fernando Fonseca. Automatic Camera Calibration Applied to Medical Endoscopy. BMVC 2009 - 20th British Machine Vision Conference, Sep 2009, London, United Kingdom. pp.1-10, 10.5244/C.23.52 . inria-00524388

\section{HAL Id: inria-00524388 https://hal.inria.fr/inria-00524388}

Submitted on 24 May 2011

HAL is a multi-disciplinary open access archive for the deposit and dissemination of scientific research documents, whether they are published or not. The documents may come from teaching and research institutions in France or abroad, or from public or private research centers.
L'archive ouverte pluridisciplinaire HAL, est destinée au dépôt et à la diffusion de documents scientifiques de niveau recherche, publiés ou non, émanant des établissements d'enseignement et de recherche français ou étrangers, des laboratoires publics ou privés. 


\title{
Automatic Camera Calibration Applied to Medical Endoscopy
}

Joao P. Barreto ${ }^{1}$

http://www.deec.uc.pt/ jpbar

Jose Roquette ${ }^{1}$

http://www.isr.uc.pt

Peter Sturm ${ }^{2}$

http://perception.inrialpes.fr/people/Sturm/

Fernando Fonseca ${ }^{3}$

fmpfonseca@fmed.uc.pt
${ }^{1}$ Institute for Systems and Robotics, Faculty of Science and Technology, University of Coimbra. 3030 Coimbra, Portugal

2 INRIA Rhone-Alpes, 38330 Montbonnot, France

${ }^{3}$ Hospitais da Universidade de Coimbra Faculty of Medicine, University of Coimbra 3000 Coimbra, Portugal

\begin{abstract}
The paper proposes a new calibration algorithm for cameras with lens distortion, that uses a single image of a planar chessboard pattern acquired in general position. The radial distortion is modeled using the first order division model, and the method provides a closed form estimation of the intrinsic parameters and distortion coefficient. The experimental evaluation shows that the calibration accuracy is comparable to state-of-the-art algorithms requiring multiple input images. We believe that our approach is particularly well suited for the the calibration of medical endoscopes in computer aided surgery. Since the lens is mounted on the camera before each usage in the OR, the calibration procedure must be performed by the clinical practitioner with minimum effort. We solve this problem by proposing a fully automatic procedure that requires no human intervention other than acquiring a single calibration image ${ }^{1}$.
\end{abstract}

\section{Introduction}

Endoscopes enable minimally invasive medical procedures with little or no injury to healthy organs and tissues. Most of these procedures are very difficult to execute, and even the best trained professionals make mistakes with inevitable consequences for the patient. In the last decade efforts have been done towards developing systems for computer aided surgery [2]. The idea is to assist the practitioner during the intervention in order to minimize human error and improve clinical results. Many of these systems rely in the processing of endoscopic video to increase surgeon's perception of the scene [8], and provide guidance inside the human body [7].

Camera calibration can hardly be avoided in the context of image-based computer aided surgery. However, developing an effective method for geometric calibration of a medical endoscope is a very challenging task [14]. The reasons are the following: (i) in general 
the calibration result must be extremely accurate, because many medical procedures require positioning accuracies of the order of tenth of millimeter; (ii) the endoscope optics introduces strong radial distortion that must be considered in the projection model; (iii) and finally the calibration procedure has to be performed by a non-expert in the Operation Room (OR), which requires the method to be simple, fast and robust.

Geometric camera calibration is a well studied topic, and currently several methods and software are available for accomplishing the task. Bouguet's implementation of Zhang's method for calibrating a camera from a minimum of three grid images is particularly popular $[3,15]$. The toolbox is also able to accommodate non-linear lens distortions by considering a 7th order polynomial model in a global iterative optimization step [5]. Unfortunately the necessary calibration procedure does not meet the usability requirements specified above. Camera calibration using the Bouguet toolbox involves the acquisition of several grid images and the manual selection of corner points. This selection is usually problematic in the presence of strong lens distortion, with the user being asked to provide a rough initialization of the relevant parameters. We can hardly imagine a medical doctor being eager to acquire 10 to 20 grid images during his regular clinical practice, and then struggle to manually select points over all this imagery. Not solving this usability issue can potentially condemn to failure any kind of assistive technology relying on endoscopic video processing. Wengert et al. address the problem by proposing an add-on to the Bouguet toolbox, where a dot grid is used to avoid manual point selection [14]. Unfortunately, and since it is impossible to determine the centers of the dots in an image with radial distortion, replacing the chessboard by dot grid implies a decrease in the calibration accuracy.

This article proposes a method for endoscope calibration that overcomes the usability issues without compromising the accuracy. The approach is fully automatic and, to the best of our knowledge, it is the first algorithm in the literature able to calibrate a camera with radial distortion from a single image of a planar grid. We build on recent developments in using lifted coordinates to model the projection in central catadioptric systems [12]. This theory is extended to the case of medical endoscopes by exploring the similarities between the division model for radial distortion and the para-catadioptric projection [1]. The experimental results show that our linear calibration method from a single image presents an accuracy comparable with Bouguet, that uses multiple images and iterative non-linear optimization.

Notation: We do not distinguish between a projective transformation and the matrix representing it. Matrices are represented by symbols in sans serif font, e.g. $M$, and vectors by bold symbols, e.g. Q. Equality of matrices or vectors up to a scalar factor is written as $\sim$. By default points and conics are represented in homogeneous coordinates.

\section{Camera Model}

The images provided by medical endoscopes present strong radial distortion with straight lines in the scene being projected into curves in the image. In the last century different authors have proposed different models for radial lens distortion $[5,6]$. This article assumes that the endoscope follows the first order division model proposed in $[4,9]$. 


\subsection{Projection using the Division Model for Lens Distortion}

The division model maps undistorted points $\mathbf{u}$ into distorted points $\mathbf{d}$ as follows [1]:

$$
\mathbf{d} \sim \Gamma_{\xi}(\mathbf{u}) \sim\left[\begin{array}{lll}
2 u_{1} & 2 u_{2} & u_{3}+\sqrt{u_{3}^{2}-4 \xi\left(u_{1}^{2}+u_{2}^{2}\right)}
\end{array}\right]^{\top}
$$

$\Gamma_{\xi}$ is a function in the projective plane $\mathscr{P}^{2}$, and $\xi$ is a negative parameter that accounts for the amount of radial distortion. Let $\mathbf{Q}$ be the non-homogeneous coordinates of a 3D point with respect to an arbitrary world reference frame. $\mathbf{Q}$ is projected by the camera with lens distortion into the image point

$$
\mathbf{q} \sim \mathrm{K} \Gamma_{\xi}(\underbrace{\left[\begin{array}{ll}
\mathrm{R} & \mathbf{t}
\end{array}\right]}_{\mathrm{P}}\left[\begin{array}{c}
\mathbf{Q} \\
1
\end{array}\right])
$$

$\mathrm{P}$ is a $3 \times 4$ projection matrix depending on the camera rotation $\mathrm{R}$ and translation $\mathbf{t}$ [11]. $\mathrm{K}$ stands for the matrix of intrinsic parameters, with $f$ being the focal length, $a$ the aspect ratio, $s$ the skew, and $\left(c_{x}, c_{y}\right)$ the principal point.

$$
\mathrm{K} \sim\left[\begin{array}{ccc}
a f & s f & c_{x} \\
0 & a^{-1} f & c_{y} \\
0 & 0 & 1
\end{array}\right]
$$

Let's assume that the imaged 3D points lie on a plane $\Pi$ and, without loss of generality, consider that the world reference frame is aligned with $\Pi$ such that $\Pi \sim\left[\begin{array}{llll}0 & 0 & 1 & 0\end{array}\right]^{\top}$. Under such conditions the projection matrix $P$ is replaced by a $3 \times 3$ plane-to-image homography $H$, which encodes the translation $\mathbf{t}$ and the two first columns of the rotation $R$ [11]. A point in the 3D plane, with local homogeneous coordinates $\mathbf{g}$, is projected at

$$
\mathbf{q} \sim \mathrm{K} \Gamma \xi(\underbrace{\left[\begin{array}{lll}
\mathbf{r}_{1} & \mathbf{r}_{2} & \mathbf{t}
\end{array}\right]}_{\mathrm{H}} \mathbf{g})
$$

\subsection{Projection using Lifted Coordinates}

The projection equations derived above are non-linear in homogeneous coordinates because of the radial distortion function $\Gamma_{\xi}$. This section shows how they can be written in a linear manner using the so called lifted coordinates [12]. As pointed out by Barreto in [1], the division model for lens distortion is isomorphic to projecting the $3 \mathrm{D}$ points $\mathbf{Q}$ on an parabolic surface, and imaging the paraboloid by a pin-hole camera placed at its vertex. The paraboloid model relates with cameras with lens distortion as the sphere model relates with central catadiotpric systems [10]. Fig. 1(a) schematizes the model where $\mathbf{O}$ is the origin of the camera reference frame (the effective viewpoint). The pin-hole camera is placed at the vertex $\mathbf{C}$ with its optical axis aligned with the symmetry axis of the paraboloid. The vertex is displaced with respect to $\mathbf{O}$ by a negative distance $\xi$ that accounts for the amount of radial distortion.

Let $\mathbf{Q}$ be the 3D non-homogeneous coordinates of a point in the camera reference frame. Line $\mathbf{L}$, defined by $\mathbf{Q}$ and the effective viewpoint $\mathbf{O}$, intersects the paraboloid in two distinct points, which are imaged at locations $\mathbf{q}_{+}$and $\mathbf{q}_{-}$. The former is the 'real' image of $\mathbf{Q}$ $\left(\mathbf{q} \sim \mathbf{q}_{+}\right)$, while the latter is a 'virtual' image henceforth referred as the antipodal image 


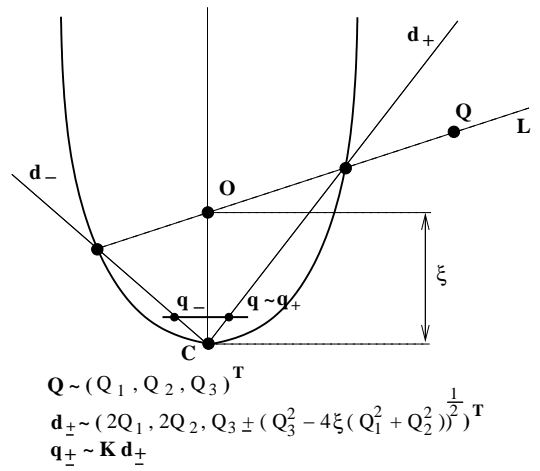

(a) Paraboloid Model.

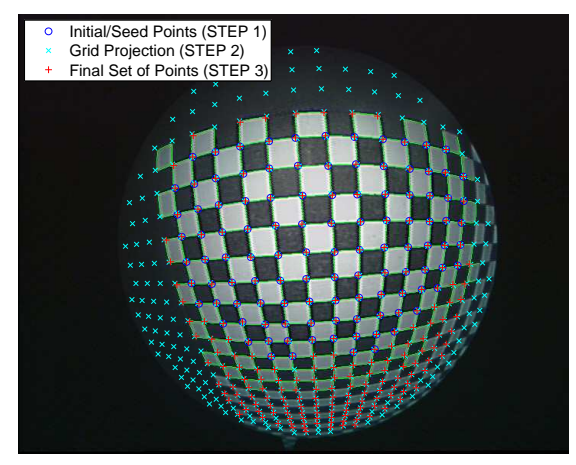

(b) Test Image 1

Figure 1: The left side is a scheme of the paraboloid model for cameras with lens distortion proposed in [1]. The right side shows one of the test images used in the experimental evaluation of section 4 . The marked points are the outcome of steps 1, 2 and 3 of the algorithm outlined in section 3.3. These steps concern the automatic detection of plane-image correspondences.

point. Thus, $\mathbf{Q}$ has two images $\mathbf{q}_{+}$and $\mathbf{q}_{-}$that are algebraically coupled. Consider the projective points $\mathbf{d}_{ \pm} \sim \mathrm{K}^{-1} \mathbf{q}_{ \pm}$. As suggested by Sturm in [12], a pair of points can be represented by a rank 2 dual conic $\Omega$.

$$
\Omega \sim \mathbf{d}_{+} \mathbf{d}_{-}^{\top}+\mathbf{d}_{-} \mathbf{d}_{+}^{\top} \sim\left[\begin{array}{ccc}
Q_{1}^{2} & Q_{1} Q_{2} & \frac{1}{2} Q_{1} Q_{3} \\
Q_{1} Q_{2} & Q_{2}^{2} & \frac{1}{2} Q_{2} Q_{3} \\
\frac{1}{2} Q_{1} Q_{3} & \frac{1}{2} Q_{2} Q_{3} & 4 \xi\left(Q_{1}^{2}+Q_{2}^{2}\right)
\end{array}\right]
$$

Since $\Omega$ is a $3 \times 3$ symmetric matrix with 6 distinct entries, it can be uniquely represented by a $6 \times 1$ homogeneous vector. This vector stacks the upper triangular part of the matrix and can be computed using the $v_{\text {sym }}$ operator

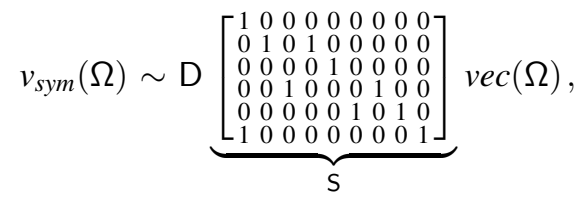

with $\operatorname{vec}(\Omega)$ being the column-wise vectorization of $\Omega, \mathrm{S}$ a permutation matrix that adds repeated entries in $\operatorname{vec}(\Omega)$, and D a diagonal matrix such that $D_{i i}=\sum_{j=1}^{9} S_{i j}$. Since $\Omega$ depends on the second order monomials of $\mathbf{Q}$, equation 2 can be written as a linear transformation of the vector of lifted coordinates $\widehat{\mathbf{Q}} \sim v_{\text {sym }}\left(\mathbf{Q} \mathbf{Q}^{\top}\right)$. It follows that

$$
v_{\text {sym }}\left(\mathbf{d}_{+} \mathbf{d}_{-}^{\top}+\mathbf{d}_{-} \mathbf{d}_{+}^{\top}\right) \sim \underbrace{\left[\begin{array}{cccccc}
1 & 0 & 0 & 0 & 0 & 0 \\
0 & 1 & 0 & 0 & 0 & 0 \\
0 & 0 & 1 & 0 & 0 & 0 \\
0 & 0 & 0 & \frac{1}{2} & 0 & 0 \\
0 & 0 & 0 & 0 & \frac{1}{2} & 0 \\
\xi & 0 & \xi & 0 & 0 & 0
\end{array}\right]}_{\mathbf{X}_{\xi}} \widehat{\mathbf{Q}} .
$$

Let's return to the case of projecting points $\mathbf{g}$ lying on a 3D plane $\Pi$ (equation 1). Taking into account that $\mathbf{q}_{ \pm} \sim \mathrm{K} \mathbf{d}_{ \pm}$, and that the homography $\mathrm{H}$ maps points $\mathbf{g}$ in plane coordinates, 
into points $\mathbf{Q}$ in camera coordinates $(\mathbf{Q} \sim \mathbf{H} \mathbf{g})$, it arises that

$$
v_{\text {sym }}\left(\mathbf{q}_{+} \mathbf{q}_{-}^{\top}+\mathbf{q}_{-} \mathbf{q}_{+}^{\top}\right) \sim \underbrace{\widehat{\mathrm{K}} \mathbf{X}_{\xi}}_{\mathrm{H}_{\text {dist }}} \widehat{\mathrm{H}} \widehat{\mathbf{g}},
$$

with $\widehat{K}$ and $\widehat{H}$ being the lifted representations of $\mathrm{K}$ and $\mathrm{H}$. The lifted representation of a generic $3 \times 3$ matrix $A$ is given by the following formula, where $\otimes$ denotes the Kronecker product [12]

$$
\widehat{A} \sim D^{-1} S(A \otimes A) S
$$

\section{Calibration from a Single Plane-to-Image Homography}

Equation 3 shows that the projection of plane by a camera with lens distortion can be expressed by a $6 \times 6$ matrix $\mathrm{H}_{\text {dist }}$. The lifted homography $\mathrm{H}_{\text {dist }}$ is a singular matrix $\left(\mathrm{X}_{\xi}\right.$ has rank 5) that encodes the camera calibration ( $\mathrm{K}$ and $\xi$ ), as well as the relative pose between the camera and the plane $(H)$. This section shows that, while perspective camera calibration requires a minimum of three plane homographies [15], cameras with radial distortion can be fully calibrated from a single homography $\mathrm{H}_{\text {dist }}$.

\subsection{DLT Estimation of $\mathrm{H}_{\text {dist }}$}

The homography $\mathrm{H}_{\text {dist }}$ maps lifted point coordinates $\widehat{\mathbf{g}}$ into pairs of antipodal image points $\mathbf{q}_{ \pm}$. When calibrating from a grid image, we know the point in the plane $\mathbf{g}$, and and the position $\mathbf{q} \sim \mathbf{q}_{+}$where $\mathbf{g}$ is imaged. In general the 'virtual' image point $\mathbf{q}_{-}$is unknown, which precludes the direct estimation of $\mathrm{H}_{\text {dist }}$ following equation 3. Fortunately Sturm shows in [12] that $v_{\text {sym }}\left(\mathbf{q}_{+} \mathbf{q}_{-}^{\top}+\mathbf{q}_{-} \mathbf{q}_{+}^{\top}\right)$ is always in the null space of the lifted skew-symmetric matrix $\widehat{[\mathbf{q}]_{\times}}$. Thus, $\mathrm{H}_{\text {dist }}$ can be estimated by considering the following relation that arises from equation 3

$$
\widehat{[\mathbf{q}}_{\times} \mathrm{H}_{\text {dist }} \widehat{\mathbf{g}}=0 .
$$

Since matrix $\widehat{[\mathbf{q}]_{\times}}$is rank 3 , each plane-image correspondence imposes 3 independent linear constrains in the 36 entries of $\mathrm{H}_{\text {dist }}$. Therefore the lifted homography can be estimated from a minimum of 12 correspondences using a standard DLT-like approach.

\subsection{Camera Calibration by Considering the Null Space of $\mathrm{H}_{\text {dist }}$}

Consider points $\mathbf{d}_{ \pm} \sim \mathrm{K}^{-1} \mathbf{q}_{ \pm}$. From the second equation of Fig. 1(a) it follows that any pair $\mathbf{d}_{+}$and $\mathbf{d}_{-}$is harmonic with respect to the circle of radius $\xi^{-1}$. Thus, any pair of antipodal image points $\mathbf{q}_{ \pm}$must be harmonic with respect to the conic $\Omega_{\xi}$.

$$
\Omega_{\xi} \sim \mathrm{K}^{-\mathrm{T}}\left[\begin{array}{ccc}
-\xi & 0 & 0 \\
0 & -\xi & 0 \\
0 & 0 & 1
\end{array}\right] \mathrm{K}^{-1}
$$

Moreover the equation $\mathbf{q}_{-}^{\top} \Omega_{\xi} \mathbf{q}_{+}=0$ can be re-written using lifted vector representation

$$
v_{s y m}\left(\Omega_{\xi}\right)^{\top} \mathrm{D} v_{\text {sym }}\left(\mathbf{q}_{+} \mathbf{q}_{-}^{\top}+\mathbf{q}_{-} \mathbf{q}_{+}^{\top}\right)=0 .
$$


Let's return to the homographic relation of equation 3. The left multiplication by $v_{s y m}\left(\Omega_{\xi}\right)^{\top} \mathrm{D}$ must zero both sides of the equation. This means that $v_{s y m}\left(\Omega_{\xi}\right)$ can be computed from the left null space of $\mathrm{H}_{\text {dist }}$ as shown below

$$
v_{\text {sym }}\left(\Omega_{\xi}\right) \sim \mathrm{D}^{-1} \mathscr{N}\left(\mathrm{H}_{\text {dist }}^{\top}\right)
$$

By considering the Cholesky decomposition of $\Omega_{\xi}$ we obtain

$$
\mathrm{K}_{\eta} \sim\left[\begin{array}{ccc}
a \eta & s \eta & c_{x} \\
0 & a^{-1} \eta & c_{y} \\
0 & 0 & 1
\end{array}\right] \quad \text { with } \quad \eta=\frac{f}{\sqrt{-\xi}}
$$

Remark that $\mathrm{K}_{\eta}$ is such that $\Omega_{\xi}=\mathrm{K}_{\eta}^{-\mathrm{T}} \mathrm{K}_{\eta}^{-1}$. This matrix directly encodes every calibration parameter with the exception of the focal length and the distortion coefficient. Parameters $f$ and $\xi$ define the ratio $\eta$, but decoupling them requires inverting the plane-to-image homography. It can be shown that the following relation holds (the proof is omitted because of space limitations).

$$
\mathbf{g} \sim \mathrm{H}^{-1} \underbrace{\left[\begin{array}{ccc}
1 & 0 & 0 \\
0 & 1 & 0 \\
0 & 0 & \sqrt{-\xi}
\end{array}\right]}_{\mathrm{G}_{\xi}} \underbrace{\left[\begin{array}{cccccc}
0 & 0 & 0 & 1 & 0 & 0 \\
0 & 0 & 0 & 0 & 1 & 0 \\
-1 & 0 & -1 & 0 & 0 & 1
\end{array}\right]}_{\mathrm{B}} \widehat{\mathrm{K}}_{\xi} \widehat{\mathbf{q}}
$$

Each image point $\mathbf{q}$ can be mapped into a direction $\mathbf{b} \sim B \widehat{K}_{\xi} \widehat{\mathbf{q}}$. $\mathbf{b}$ is the back-projection direction of $\mathbf{q}$ up to a similarity transformation $G_{\xi}$ [11]. Let's use the correspondences $\mathbf{b}-\mathbf{g}$ to estimate the matrix product $\mathrm{H}^{-1} \mathrm{G}_{\xi}$, and factorize the similarity transformation $\mathrm{G}_{\xi}$ from the result following the approach suggested in [13]. The distortion parameter $\xi$ can now be determined from $\mathrm{G}_{\xi}$, and the focal length can be computed as $f=\eta \sqrt{-\xi}$.

\subsection{Algorithm for Single Image Calibration (SIC)}

This section outlines our algorithm for calibrating a medical endoscope from a single image of a planar grid. The method is fully automatic and requires no human intervention for detecting the correspondences between image and plane. It is assumed that the calibration plane is in the image foreground (see Figures 1(b) and 3(a)), that the grid is regular with known dimensions, and that lens distortion follows the division model.

1. Initial detection of plane-to-image correspondences (seed points): Radial distortion avoids the accurate detection of corners in the image periphery (see Fig. 1(b)). We start by establishing an initial mask using conservative edge detection with a high threshold. Corners over the mask are detected as local maxima in the entropy of the image gradient. Square regions are segmented and counted to match the detected corners with the corresponding grid points.

2. Projection of the grid into the image using an initial estimate of $\mathrm{H}_{\text {dist }}$ : The detected plane-to-image correspondences enable an initial DLT estimation of $\mathrm{H}_{\text {dist }}$ (section 3.1). The result is used to project an augmented grid into the image (Fig. 1(b)).

3. Harris corner detection: Many points of the projected grid do not correspond to a real image corners. Standard corner detection, with a small search window, is used to discard wrong candidates and refine the position of valid ones (Fig. 1(b)). 


\begin{tabular}{|c||c|c|c||c|c|c|c|}
\hline & $\eta$ & $\mathbf{f}$ & $\xi$ & $\mathbf{a}$ & $\mathbf{s}$ & $\mathbf{c}_{x}$ & $\mathbf{c}_{y}$ \\
\hline \hline Mean Value & 438.85 & 301.34 & -0.47 & 0.998 & $-4.1 e-4$ & 375.72 & 317.29 \\
Standard Deviation & 8.73 & 26.88 & 0.08 & $1.3 e-3$ & $2.4 e-3$ & 3.34 & 7.18 \\
\hline \hline
\end{tabular}

Bouguet Value

300.82

0.984

$2.2 e-3$

371.70318 .95

Table 1: Mean and standard deviation of the calibration parameters across the 12 test images.

4. Final estimation of the homography $\mathrm{H}_{\text {dist }}$ : The homography is re-estimated from the entire set of correct plane-to-image correspondences (section 3.1).

5. Computation of $\Omega_{\xi}$ and $\mathrm{K}_{\xi}: \Omega_{\xi}$ is computed from the left null space of $\mathrm{H}_{\text {dist }}$ (equation 4 ), and $\mathrm{K}_{\xi}$ is determined by Cholesky decomposition. The aspect ratio $a$, skew $s$, and principal point $\left(c_{x}, c_{y}\right)$ are directly recovered from $\mathrm{K}_{\xi}$ (equation 5).

6. Decoupling of the focal length $f$ from the distortion parameter $\xi$ : The similarity transformation $\mathrm{G}_{\xi}$ is estimated after back-projecting the image points. $\xi$ and $f$ are computed from $\mathrm{G}_{\xi}$ and $\eta$ as explained at the end of section 3.2.

\section{Experimental Results}

The performance of the SIC algorithm was evaluated using a set of 12 images acquired by an off-the-shelf medical arthroscope. Two of these images are exhibited in Figures 1(b) and $3(a)$, where the grid is viewed from two substantially different viewpoints. The calibration procedure is independently run for each test image. Fig. 2(a) shows the outcome of steps 1 to 3, which concern the automatic detection of plane-image correspondences. The number of detections varies across examples, with images 11 and 12 having the minimum number of initial points required for the homography estimation. Despite the input variability the calibration results present good repeatability across test images (see Fig. 2(b)(c) and Tab. 1). The larger standard deviations were observed for parameters $f$ and $\xi$. This is explained by the singularity in estimating $\mathrm{G}_{\xi}$ (step 6 of the procedure) when the calibration plane is fronto-parallel to the camera [15]. Thus, in some examples the decoupling between $f$ and $\xi$ tends to be ill-conditioned. This is also the reason explaining the poor results in recovering $f$ from image 1(Fig. 2(c)).

In addition to applying our method to each image, we also calibrated the arthroscope using the Bouguet toolbox [3]. This toolbox implements Zhang's method [15], and it is probably the state-of-the-art software for camera calibration. The cameras was calibrated using manually selected points in the 12 test images. The radial distortion was modeled using a 7th order polynomial model [5] with the coefficients being estimated in a final global optimization step. As shown in Figures 2 and 3 the calibration results achieved by the SIC algorithm are comparable with the ones obtained with Bouguet.

\section{Conclusions and Future Work}

We proposed the first algorithm in the literature able to calibrate a camera with radial distortion from a single image of a planar grid in general position. Our method is fully automatic, which makes it particularly relevant for computer aided medicine relying in the processing of endoscopic imagery. For these applications the endoscope has to be calibrated by the clinical 

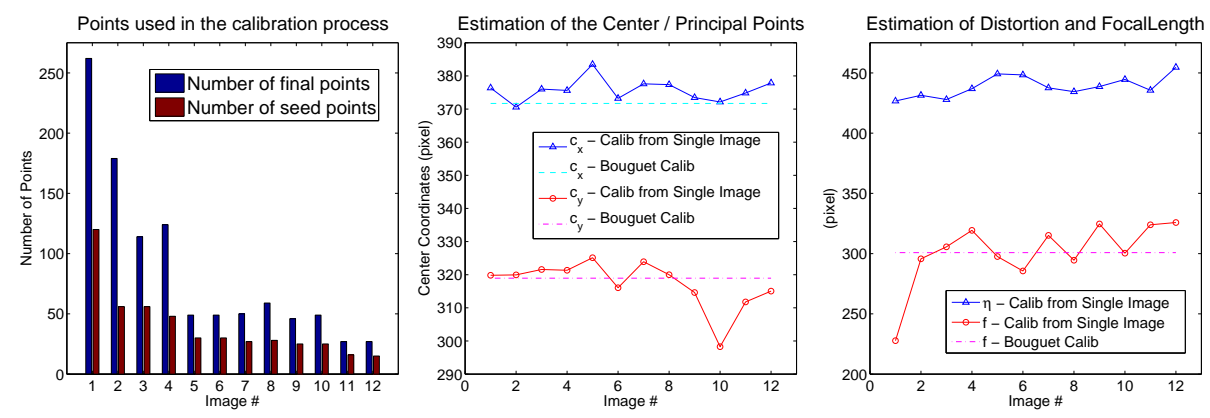

Figure 2: Single Image Calibration (SIC) applied to 12 test images. The graphic on the left shows for each example the plane-image correspondences detected at the end of steps 1 (seed points) and 3 (final points). The graphic in the middle concerns the results in estimating the principal point $\left(c_{x}, c_{y}\right)$. The right graphic shows the estimated $\eta$ and focal length $f$. The figure compares the results of 12 independent calibrations using SIC (each image enables a full calibration), against the result obtained with Bouguet's that simultaneously uses the 12 images and performs final global refinement with iterative optimization.

practitioner in its environment, which implies a minimal effort procedure. We believe that our solution is the first one meeting such requirements without compromising the calibration accuracy. We are currently running clinical trials to better assess the usability of method.

The experiments show that our single image calibration has good repeatability and provides results comparable to the ones obtained with the Bouguet toolbox [3]. As future work we intend to improve the accuracy of our calibration by refining the parameters in a complementary non-linear optimization step. This step will also enable using higher order models that better accommodate certain types of distortions.

\section{Acknowledgment}

Joao P. Barreto, Jose Roquette and Fernando Fonseca are grateful to the Portuguese Science Foundation for generous funding through grant PTDC/EEA-ACR/68887/2006. Joao P. Barreto also acknowledges the Portuguese Agency for Innovation and QREN for support through grant Ver+Saude: RDFixer.

\section{References}

[1] J. P. Barreto. A unifying geometric representation for central projection systems. Computer Vision and Image Understanding, 103, September 2006.

[2] Norman T. Berlinger. Robotic surgery. squeezing into tight places. New England Journal of Medicine, May 2006.

[3] Jean Yves Bouguet. Camera calibration toolbox for matlab. URL http://www . vision.caltech.edu/bouguetj/calib_doc/index.html. 


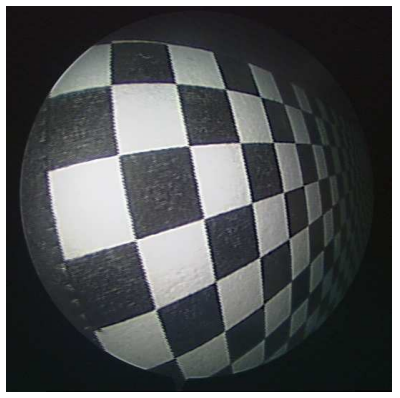

(a) Test Image 10

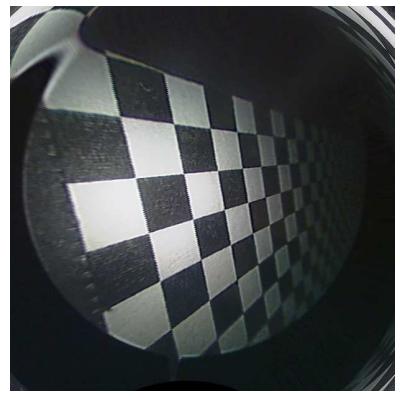

(b) Bouguet Correction

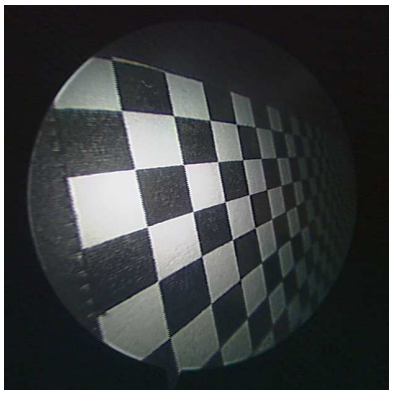

(c) SIC Correction

Figure 3: Distortion correction of test image 10: The figure compares the distortion correction using Bouguet calibration and using the single image calibration (SIC). The rectification is comparable with the lines being straight in both images. Bouguet correction tends to be marginally better, probably because it uses three distortion coefficients ( 7 th order polynomial model), while SIC assumes a simple one parameter model. The rectification with Bouguet presents some artifacts because of the non-monotonic behavior of the distortion profile in the image periphery.

[4] C. Brauer-Burchardt and K. Voss. A new algorithm to correct fish-eye- and strong wideangle-lens-distortion from single images. In IEEE Int. Conf. on Image Processing, 2001.

[5] D. C. Brown. Decentering distortion of lens. Photogrametric Engineering, 1966.

[6] A. Conrady. Decentering lens systems. Monthly Notes Royal Astronomic Society, 1919.

[7] R. Taylor D. Burschka, M. Li and G. Hager. Scale-invariant registration of monocular endoscopic images to ct-scans for sinus surgery. In Int. Conf in Medical Imaging Computing and Computer Assisted Intervention, 2004.

[8] F. Devernay, F. Mourgues, and È. Coste-Manière. Towards endoscopic augmented reality for robotically assisted minimally invasive cardiac surgery. In IEEE Int. Workshop on Medical Imaging and Augmented Reality, 2001.

[9] A. Fitzgibbon. Simultaneous linear estimation of multipleview geometry and lens distortion. In Int. Conf. on Computer Vision and Pattern Recognition, December 2001.

[10] C. Geyer and K. Daniilidis. Catadioptric projective geometry. Int. Journal Comp. Vision, 2001.

[11] R. Hartley and A. Zisserman. Multiple View Geometry in Computer Vision. Cambridge University Press, 2000.

[12] P. Sturm and J. P. Barreto. General imaging geometry for central catadioptric cameras. In Proc. of European Conf. in Computer Vision, October 2008.

[13] P. Sturm and S. Maybank. On plane-based camera calibration: a general algorithm, singularities, applications. In IEEE Int. Conf. Computer Vision and Pattern Recognition, 1999. 
[14] C. Wengert, M. Reeff, P. Cattin, and G. Székely. Fully automatic endoscope calibration for intraoperative use. In Bildverarbeitung für die Medizin, March 2006.

[15] Zhengyou Zhang. Flexible camera calibration by viewing a plane from unknown orientations. In IEEE Int. Conf. on Computer Vision, 1999. 